Chen et al., Afr J Tradit Complement Altern Med., (2017) 14 (4): 278-288

https://doi.org/10.21010/ajtcam.v14i4.31

\title{
EFFECT OF ASTRAGALOSIDE ON VITAMIN D-RECEPTOR EXPRESSION AFTER ENDOTHELIN-1-INDUCED CARDIOMYOCYTE INJURY
}

\author{
Chen yunzhi ${ }^{1,2 *}$, Chen jiaxu1, Gao jie ${ }^{2}$, Chai yihui ${ }^{2}$, Li wen ${ }^{2}$, Qin zhong ${ }^{2}$ \\ ${ }^{1}$ School of Preclinical Medicine, Beijing University of Chinese Medicine, No. 11, Beisanhuan Donglu, \\ Chaoyang,Beijing, China 100029. ${ }^{2}$ School of Preclinical Medicine, Guiyang University of Chinese Medicine, \\ No.50, Shidonglu, Guiyang, Guizhou, China 55000.

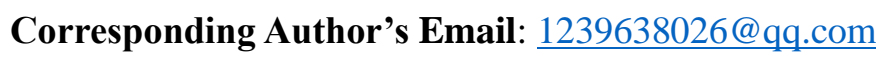

\begin{abstract}
Background: Astragaloside, which is one of the main components of Astragalus membranaceus, has been widely used in the treatment of congestive heart failure in China, and it can protect cardiomyocytes. Its mechanism of action remains unclear. Therefore, the present study was carried out to investigate the influence of astragaloside on rat cardiomyocytes stimulated with endothelin-1 (ET-1), and explored the underlying mechanism.
\end{abstract}

Materials and Methods: ET-1 was used to stimulate primary rat cardiomyocytes and establish a cardiomyocyte hypertrophy model. Different astragaloside doses were administered in combination with ET-1. Cardiomyocyte hypertrophy and apoptosis were examined using transmission electron microscopy (TEM) and flow cytometry, respectively. The molecular mechanism was explored by analyzing the mRNA of the vitamin D receptor (VDR), cytochrome P450 family 27 subfamily B member 1(CYP27B), cytochrome P450 family 24 subfamily A member 1(CYP24A) and renin mRNA levels by quantificational real-time polymerase chain reaction(qRT-PCR).

Results: Rat cardiomyocyte hypertrophy model was established successfully. Astragaloside administration significantly affected cell apoptosis and significantly inhibited ET-1-induced cardiomyocyte hypertrophy in a dose-dependent manner. Astragaloside treatment affected the expression of signaling molecules in the vitamin D axis.

Conclusion: Astragaloside inhibits ET-1-induced cardiomyocyte hypertrophy. This effect can be reversed by regulating the levels of the relevant factors in the vitamin D axis.

Key words: cardiomyocyte hypertrophy, Astragaloside, Vitamin D Receptor, renin.

\section{Introduction}

Myocardial remodeling is caused by a complex series of molecular and cellular mechanisms (Colucci, 1997), that result in an increase in cardiomyocyte diameter via broadening or linear expansion accompanied by cardiomyocyte necrosis and apoptosis (Ding et al., 2009).

Recently, many studies have demonstrated an association between the plasma vitamin D level and the occurrence of cardiovascular disease (Muscogiuri et al., 2016, Pilz et al., 2016). Vitamin D deficiency can result in activation of the renin-angiotensin-aldosterone system (RAAS)(Ferder et al., 2013). Vitamin D receptor (VDR) knockout mice and $1 \alpha$-hydroxylase knockout mice showed individual myocyte enlargement and an elevation in natriuretic peptide expression (Glenn et al., 2016). Additionally, the renin expression level is increased in systemic and cardiac RAAS activation (Xiang et al., 2005, Zhou et al., 2008). Renin mRNA and protein expression is increased in the kidneys, and the cardiomyocytes appear hypertrophy and apoptosis(Bae et al., 2013). Active vitamin D acts on the VDR to interfere with 
the function of the renin promoter and inhibit renin gene transcription activation. Thus, vitamin D can inhibit the expression of the renin gene to regulate the RAAS (Nakane et al., 2007, Li et al., 2002). Additionally, vitamin D not only inhibits the generation of proinflammatory cytokines, such as interleukin 6 (IL-6) and tumor necrosis factor $\alpha$ (TNF- $\alpha$ ), but also increases the expression of anti-inflammatory cytokines, such as interleukin 10 (IL-10) (Barker et al., 2014, Liu et al., 2011).Vitamin D also has antioxidant effects (Lee et al., 2015) that improve myocardial metabolism, protect the mitochondria (Mukherjee et al., 1981), and inhibit apoptosis to protect the myocardium (Yao et al., 2015).

Astragalus membranaceus is a commonly used traditional Chinese herb that contains flavonoids, saponins, and other active ingredients that have wide pharmacological actions, including anti-diabetic, anti-hypertensive, anti-inflammatory, and cardioprotective effects as well as the prevention of heart failure(Huang et al., 2016, Li et al., 2017).Astragaloside(AST) is one of the main components of Astragalus membranaceus which could modulate the RAAS and inhibit cardiomyocyte hypertrophy and apoptosis to protect the heart (Shi et al., 2009, Zhang et al., 2015).

Using the Arrowsmith method (http://arrowsmith.psych.uic.edu/cgi-bin/arrowsmith_uic/start.cgi), we found a correlation between Astragalus and vitamin D in the treatment of myocardial hypertrophy. Given this background, we hypothesized that the vitamin D axis played a vital role in the mechanism of astragaloside in treatment of cardiomyocyte hypertrophy. The present investigation was designed to study whether astragaloside exerted its anti-hypertrophic actions through the vitamin D axis.

\section{Materials and Methods \\ Primary culture of rat cardiomyocytes}

One-to-three-day-old newborn Sprague Dawley rats were purchased from the Animal enter of the Third Military Medical University (Chongqing, China). The ventricular myocardium was prepared as described previously (Menaouar et al., 2014). Briefly, the ventricular myocardium was excised, cut into small pieces, and washed three times with cold phosphate-buffered saline (PBS, ZSGB-BIO, China). Then, the tissues were digested 6 times for each $5 \mathrm{~min}$ at $37^{\circ} \mathrm{C}$ with $0.125 \%$ (w/v) trypsin (Beyotime, China). An equal volume of cold Dulbecco's modified Eagle's medium: Nutrient Mixture F-12 (DMEM/F12) (Gibco, USA) containing 10\% (v/v) fetal bovine serum (FBS, Gibco, USA) was added to terminate the digestion. The cells were collected and centrifuged for $10 \mathrm{~min}$ at 1000 $\mathrm{rpm}$ at room temperature. Then, the cells were collected and suspended in DMEM/F12 complete medium containing $1 \%$ antibiotics (penicillin/streptomycin, Gibco, USA) and 10\% (v/v) FBS and cultured in an incubator at $37^{\circ} \mathrm{C}$ with $5 \% \mathrm{CO}_{2}$ and saturated humidity. The culture medium was renewed after $48 \mathrm{~h}$, and the cells were cultured for an additional $24 \mathrm{~h}$. Then, the medium was changed to serum-free DMEM/F12, and the cardiomyocytes were randomly divided into 6 groups as follows: 1. Control group (PBS); 2. ET-1 (10-8 mol/L, Sigma, USA) pretreatment group; 3. VD group (ET-1, 10-8 mol/L

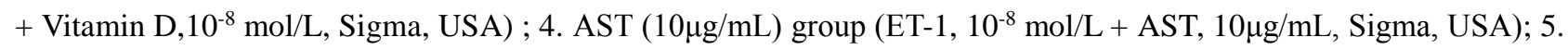
AST $(20 \mu \mathrm{g} / \mathrm{mL})$ group (ET-1, $10^{-8} \mathrm{~mol} / \mathrm{L}+\mathrm{AST}, 20 \mu \mathrm{g} / \mathrm{mL}$, Sigma, USA); and 6 . AST $(40 \mu \mathrm{g} / \mathrm{mL})$ group (ET-1,10-8 $\mathrm{mol} / \mathrm{L}+\mathrm{AST}, 40 \mu \mathrm{g} / \mathrm{mL}$, Sigma, USA). The experiments were performed after $24 \mathrm{~h}$ of treatment with the indicated drugs.

\section{Immunohistochemistry}

The cell density was adjusted to $2 \times 10^{4}$ cells $/ \mathrm{mL}$, and the cells were cultured on a cover glass. Then, the cover glass was fixed with $4 \%$ paraformaldehyde, cleared with PBS containing $0.5 \%$ Triton X-100, treated with $3 \%$ hydrogen peroxide $\left(\mathrm{H}_{2} \mathrm{O}_{2}\right)$ in methanol to quench endogenous peroxidase activity, and blocked in $6 \%$ goat serum. The samples 


\section{https://doi.org/10.21010/ajtcam.v14i4.31}

were incubated overnight with an $\alpha$-actin antibody (Abcam, USA; diluted1:200) at $4^{\circ} \mathrm{C}$. Then, the samples were incubated with a biotinylated secondary antibody (Abcam, USA; diluted 1:200) at $37^{\circ} \mathrm{C}$ for $30 \mathrm{~min}$. HRP-labeled streptavidin and the HRP substrate were added, and the samples were incubated at $37^{\circ} \mathrm{C}$ for $30 \mathrm{~min}$. A 3,3'-diaminobenzidine (DAB) (ZSGB-BIO, China) substrate system was used for color development in the dark. After counterstaining with hematoxylin, the sections were dehydrated for $5 \mathrm{~min}$, dehydrated with an alcohol gradient, cleared with xylene, and mounted with neutral mounting medium.

\section{Quantitative real-time polymerase chain reaction (qRT-PCR)}

The cells were digested and collected. RNA extraction was performed using the TRIzol reagent (Qiagen) according to the manufacturer's recommendations. Reverse transcription (RT) was conducted with random primers, and qRT-PCR was performed to examine the VDR, vitamin D 27-hydroxylase (CYP27B), vitamin D 24-hydroxylase (CYP24A) and renin mRNA expression levels; the primer sequences are listed in Table 1 . The qRT-PCR amplification conditions were as follows: $94^{\circ} \mathrm{C}$ for $4 \mathrm{~min}$, followed by 35 cycles of $94^{\circ} \mathrm{C}$ for $20 \mathrm{sec}, 60^{\circ} \mathrm{C}$ for $30 \mathrm{sec}$, and $72^{\circ} \mathrm{C}$ for $30 \mathrm{sec}$. The analysis was repeated three times for each sample.

Table 1: Primers and fluorescent probes used in the fluorescent quantitative PCR systems

\begin{tabular}{clc}
\hline Name of the primer & \multicolumn{1}{c}{ Sequence (5'-3') } & Amplified length (bp) \\
\hline VDR F & CCCGACCCTGGTGACTTTG & 188 \\
VDR R & CGGTTGTCCTTGGTGATGC & \\
CYP27B F & AGTGTTGAGATTGTACCCTGTGG & 126 \\
CYP27B R & TCCCTTGAAGTGGCATAGTGA \\
CYP24 F & ACCAAAGTGTGGCAGGCAC & \\
CYP24 R & GACAGCGGCGTACAGTTCC & 168 \\
Renin F & CTGCTCAGGCTGTTGATGGA & 168 \\
Renin R & AGTGAAAGTTGCCCTGGTAATG & \\
Actin F & CCCATCTATGAGGGTTACGC & 150 \\
Actin R & TTTAATGTCACGCACGATTTC & \\
\hline
\end{tabular}

\section{Transmission electron microscope}

The cells were collected and fixed with glutaraldehyde/osmium tetroxide fixative. The samples were cut into a series of semi-thin sections and embedded. The sections were stained with compound dye ( $0.25 \%$ sodium borate: $0.25 \%$ basic fuchsin, 1:1) and cut into ultrathin sections after observation using a microscope. The ultrathin sections were put on a copper wire mesh and attached with a film prepared using a $0.45 \%$ Fonnvar solution. Then, the samples were dried on filter paper, stained with uranyl acetate and lead citrate, and examined using a transmission electron microscope (Royal Philips Tecnai-10, the Netherlands).

\section{Flow cytometry}

The cells were harvested by enzymatic digestion and washed in PBS. A total of $3 \times 10^{5}$ cells were collected. The 


\section{https://doi.org/10.21010/ajtcam.v14i4.31}

cells were incubated with $50 \mu \mathrm{L}$ of 7 -AAD staining solution (BD) at room temperature for 10 min in the dark. The solution was then centrifuged, the supernatant was discarded, and the cells were resuspended in $450 \mu \mathrm{L}$ of binding buffer. The solution was incubated at room temperature for $10 \mathrm{~min}$ in the dark after the addition of $1 \mu \mathrm{L}$ of Annexin V-PE staining solution. Cell apoptosis was analyzed by flow cytometry (BD Influx flow cytometer, USA) after cooling the solution in an ice bath. The BD FACSTM software was used to store and retrieve the data.

\section{Statistical}

Statistical analysis of the experimental data was performed using the SPSS software 17.0 (SPSS, Chicago, IL, USA). Measurement data were presented as the mean \pm standard deviation to compare the means of two variables (not groups) for the survey data. One-way ANOVA was used to compare differences among groups. A p-value $<0.05$ was considered significant.

\section{Result}

Identification of rat cardiomyocytes

During the initial inoculation period, the primary cardiomyocytes were transparent and round. The cells adhered to the culture dish approximately $24 \mathrm{~h}$ after inoculation. Non-adherent cells and impurities were eliminated with time and medium changes. After adherence, the cells began to spread and divide. The cells were fusiform or polygonal in shape with the nucleus located in the center of the cell. Cardiomyocytes were identified by immunohistochemical staining for rat $\alpha$-actin (Fig. 1).

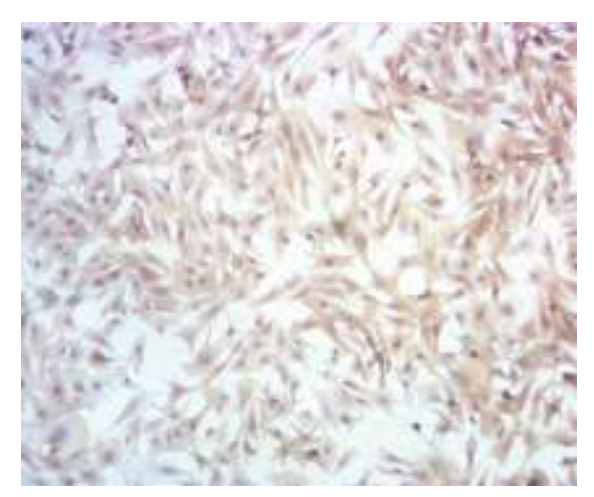

$100 \mathrm{X}$

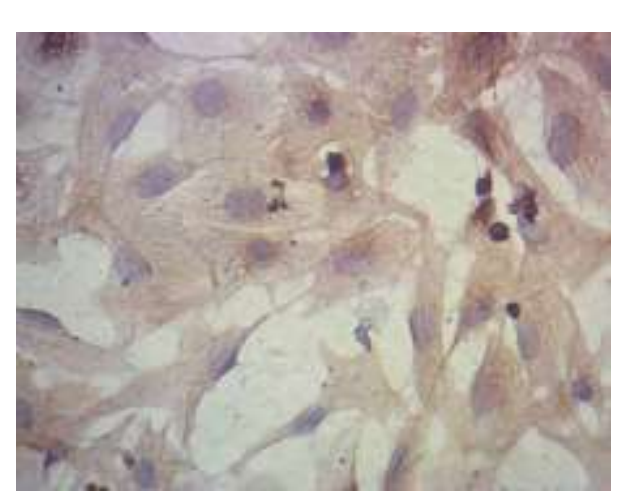

$400 \mathrm{X}$

Figure 1: Identification of primary cardiomyocytes of rats

\section{Quantitative real-time polymerase chain reaction (qRT-PCR)}

The VDR, CYP24A, CYP27B and renin mRNA expression levels were evaluated by qRT-PCR. For each gene, the primer sets amplified a unique PCR product with a single-peak dissociation curve. The amplification efficiency of the PCR reactions was higher than 95\%. Relative quantification of the PCR results indicated that the VDR and CYP27B mRNA expression levels were significantly reduced in the ET-1 group compared with the control group, whereas the CYP24A and renin mRNA expression levels were significantly increased in the ET-1 group (P<0.05). The mRNA levels of all genes in VD group were similar to the expression levels in the control group (P>0.05). AST groups had an effect similar to VD group. Thus, astragaloside and vitamin D may partially counteract the effects of ET-1 on the mRNA levels 


\section{https://doi.org/10.21010/ajtcam.v14i4.31}

of the candidate genes in a dose-dependent manner (Fig. 2).
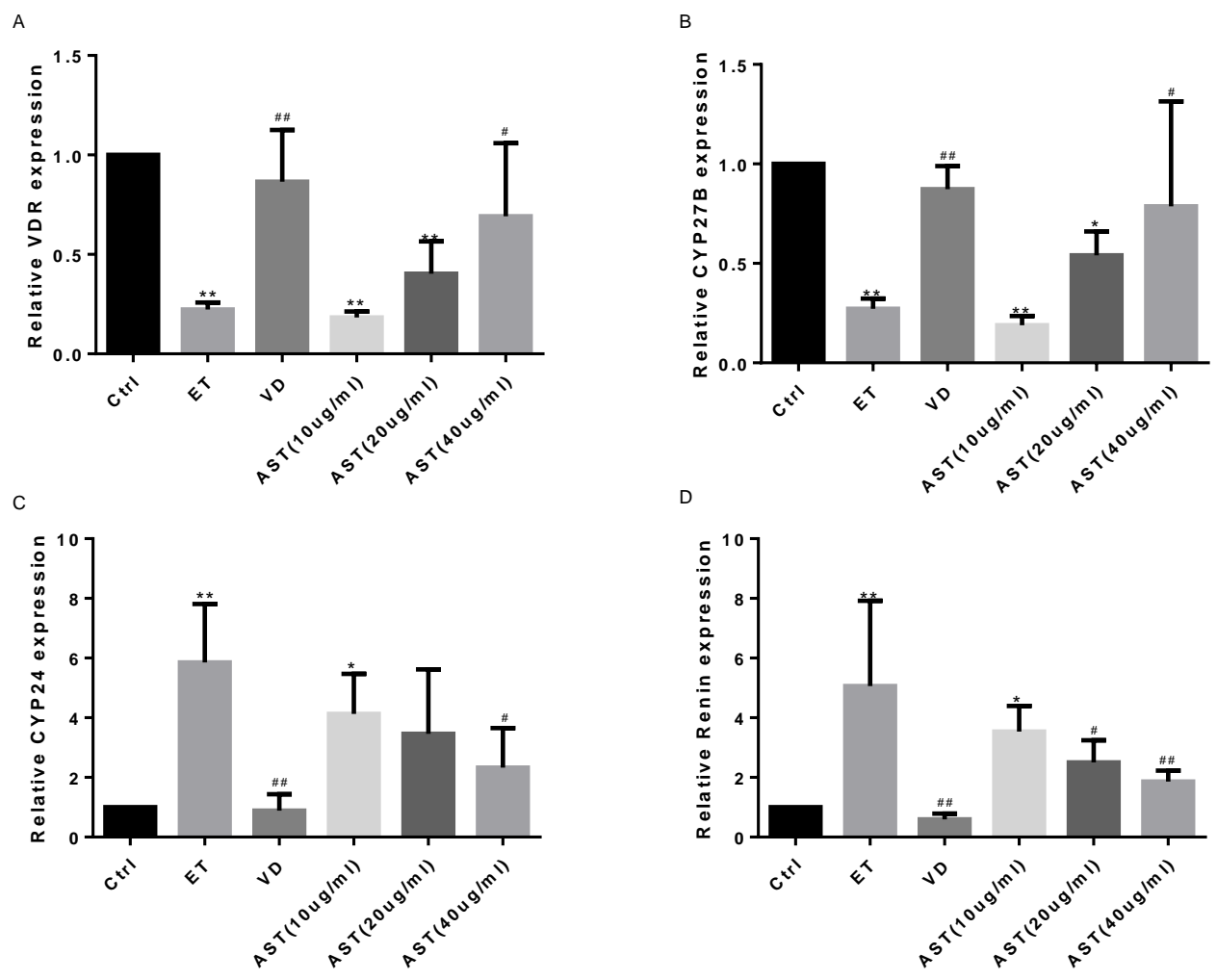

Figure 2: ET-1-induced cardiomyocyte hypertrophy was attenuated by AST in neonatal rat cardiomyocytes. (A-D) The mRNA expression levels of VDR, CYP27B, CYP24 and renin were evaluated by qRT-PCR analysis in cardiomyocytes exposed to control (PBS), ET-1 (10-8 $\mathrm{mol} / \mathrm{L})$, VD and AST $(10-40 \mu \mathrm{g} / \mathrm{mL})$. The data are expressed as the mean $\pm \mathrm{sd}$.

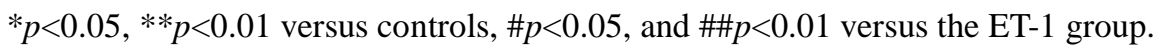

\section{Cardiomyocyte hypertrophy}

Ultrathin sections of the cells in each group were observed by TEM (Fig. 4). The observed ultrastructures revealed that the cardiomyocytes in the control group had a uniform nucleoplasm and smooth nuclear membranes. The mitochondrial cristae in the cardiomyocytes were arranged in an orderly and dense manner. After treatment with ET-1, the cells exhibited marginalized and aggregated chromatin and crinkled and distorted nuclear membranes; we also observed dense bodies in the cytoplasm. The cristae of the mitochondria were disordered, loose, and broken. When ET-1 and vitamin D were co-administered, the cells resembled the control group cells. When ET-1 and AST were co-administered, the area of the nuclei increased. The pathological changes in the mitochondria of the rat cardiomyocytes improved, and the chromatin aggregated in the middle of the nuclei (Fig. 3). 

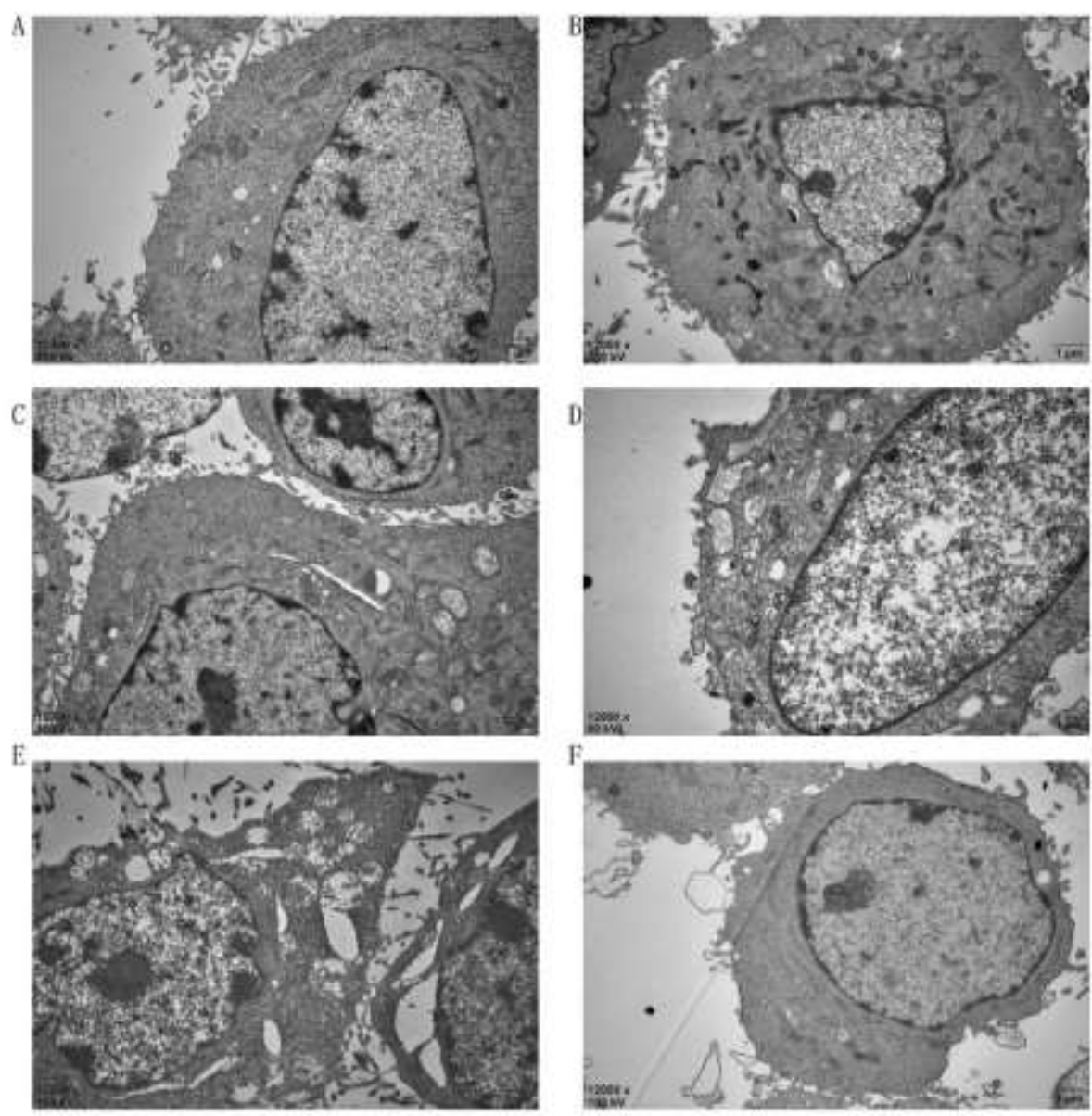

Figure 3: Astragaloside effect on mice cardiomyocyte. Transmission electron microscope. (A-F show group Ctrl, ET-1, VD, AST(10-40ug/ml))

\section{Flow cytometry}

To examine the effect of AST on ET-1-induced cell death, apoptosis was examined by flow cytometry. The flow cytometry results indicated that the cell apoptosis rates in the control group, ET-1 group, ET-1 +VD group and ET-1 + AST group were $7.45 \pm 0.13 \%, 14.41 \pm 0.11 \%, 8.3 \pm 0.44 \%, 13.35 \pm 0.27 \%, 9.77 \pm 0.07 \%$ and $8.21 \pm 0.07 \%$, respectively. A significant difference was observed in the survival rate between the control group and each experimental group $(\mathrm{P}<0.01)$ (Fig. 4). 
Chen et al., Afr J Tradit Complement Altern Med., (2017) 14 (4): 278-288 https://doi.org/10.21010/ajtcam.v14i4.31

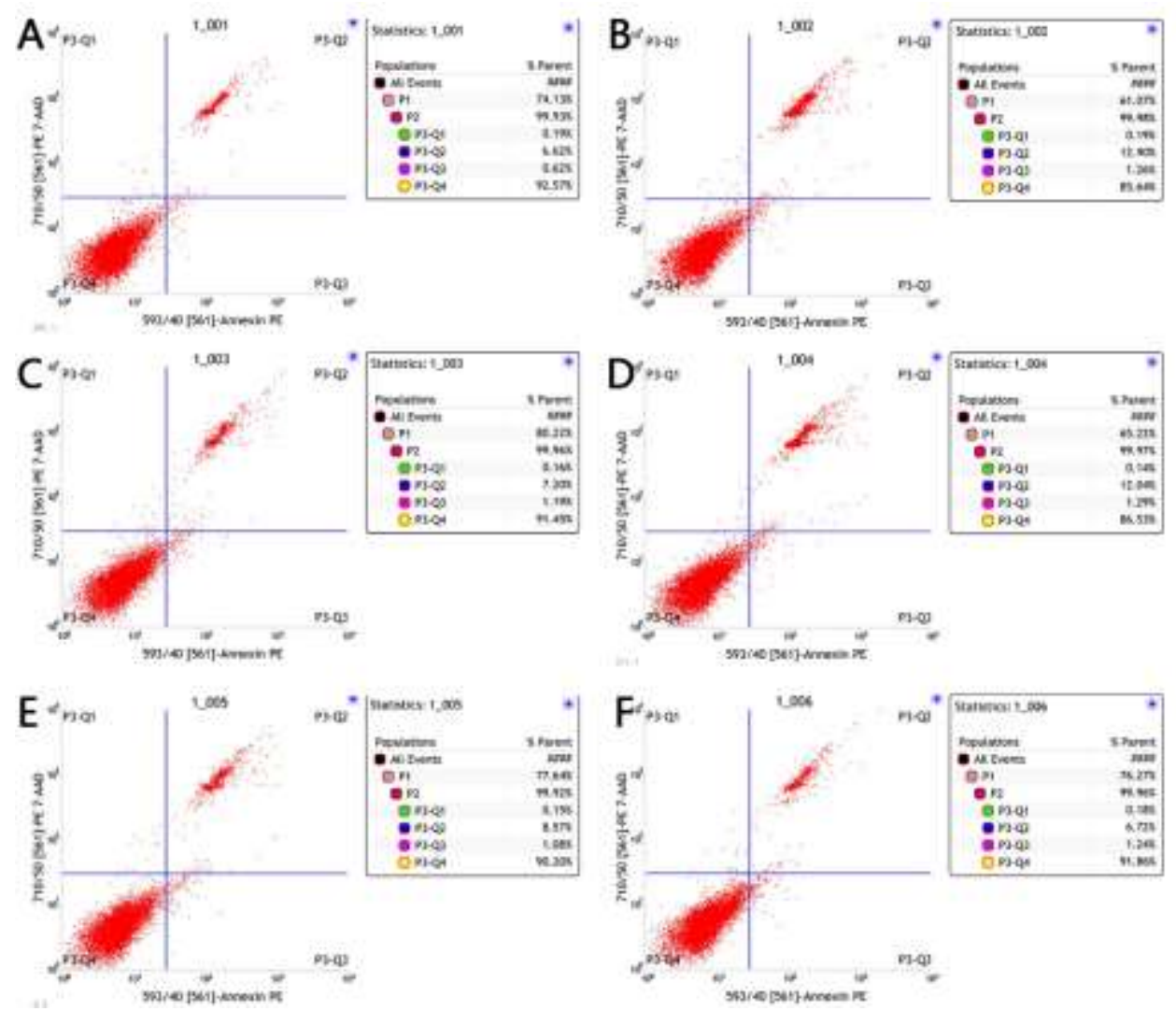

G

m yocyte apoptosis

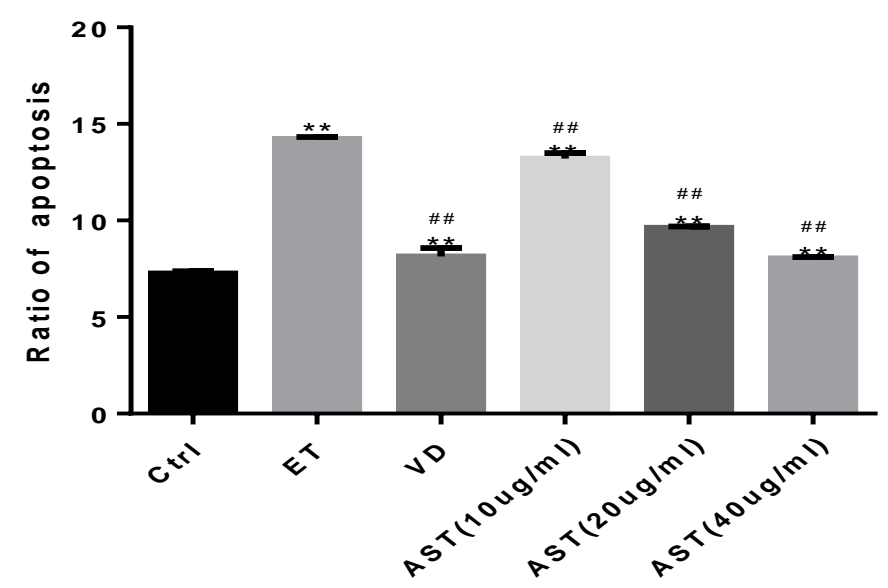

Figure 4: Effect of AST on ET-1-induced cell apoptosis.(A-F show group Ctrl, ET-1, VD, AST(10-40ug/ml)) .Figure G shows the rate of cardiomyocyte apoptosis. Data are expressed as means $\pm \mathrm{sd}$. $* p<0.05, * * p<0.01$ versus control, \#p< 0.05, \#\#p 0.01 versus ET-1 group. 


\section{Discussion}

Cardiomyocyte hypertrophy is an adaptive response to a variety of cardiovascular stimuli, such as the hemodynamic load (Devereux et al., 1983), growth factors(Karpanen et al., 2008) and hormones(Hu et al., 2005). Chronic cardiomyocyte hypertrophy may lead to cardiac arrhythmia (Ehses et al., 2000), myocardial infarction, heart failure, and even death; therefore, cardiomyocyte hypertrophy is considered an independent risk factor for an increase in cardiovascular morbidity and mortality(Mahdy Ali et al., 2012).

Renin is a rate-limiting step in angiotensin II (Ang II ) production. Renin not only initiates the RAAS chain reaction with angiotensinogen (Yoshida et al., 2014), but also activates the prorenin receptor-activated intracellular signal transduction pathway, which is dependent on Ang II production. Thus, renin plays a vital role in promoting inflammation and proliferation, leading to cardiomyocyte hypertrophy and hyperplasia(Saris et al., 2006). A large number of studies have confirmed that activated RAAS regulates cardiomyocyte growth and apoptosis by endocrine and paracrine effects (Weir and Dzau, 1999)and that activate proinflammatory cytokines and exacerbate the inflammatory response (Sciarretta et al., 2009). Angiotensin converting enzyme inhibitors (ACEIs) and angiotensin II type 1 receptor blockers (ARBs) play important roles in the treatment of chronic heart failure and coronary artery disease (Cohn, 2007, Bavry et al., 2014). However, these drugs failed to elicit the desired results (Lother and Hein, 2016). Currently, an emerging field is seeking to directly regulate the synthesis and release of renin through endogenous metabolic intermediates that block RAAS from the source. Vitamin D and it's analogues may be novel drugs for the prevention and treatment of chronic cardiovascular disease via the inhibition of the secretion of renin and the systemic and local RAAS(Pourdjabbar et al., 2013).

Astragaloside has wide pharmacological actions, including anti-diabetes, anti-hypertension, anti-inflammation (Xie and $\mathrm{Du}, 2011$, Wang et al., 2016), cardiomyocyte protection, and anti-heart failure (Luo et al., 1995). Astragaloside regulates cardiomyocytes mainly by inducing NO release, preventing lipid peroxidation (Wang et al., 2015), eliminating oxygen free radicals, reducing oxidative damage in the body, improving the vigor of the myocardial sarcoplasmic reticulum $\mathrm{Ca}^{2+}$-ATPase (SERCA), stabilizing the intracellular $\mathrm{Ca}^{2+}$ concentration, inhibiting the overload of intracellular calcium(Meng et al., 2005, Lu et al., 1999), reducing cell damage, activating mitochondrial apoptotic pathways, and inhibiting cell apoptosis (Guan et al., 2015). Regulating the excessive activation of the RAAS and cytokine production may represent major roles in the inhibition of cardiomyocyte hypertrophy (Shi et al., 2009, Yang et al., 2013).

The preliminary results of the present study indicated that astragaloside inhibited ET-1-induced cardiomyocyte hypertrophy. The flow cytometry results showed that ET-1 induced apoptosis in cardiomyocytes and that astragaloside and vitamin D significantly ameliorated ET-1-induced cardiomyocyte apoptosis. Astragaloside influenced the VDR, CYP27B, and CYP24A mRNA expression levels in a dose-dependent manner. This result suggests that astragaloside treatment may reverse cardiomyocyte hypertrophy by regulating the levels of relevant factors in the vitamin $\mathrm{D}$ axis.

In summary, our study demonstrated that astragaloside was cardioprotective in cultured cardiomyocytes and exerted significant anti-hypertrophic activities at least partially through the vitamin D axis. These findings suggest that uncovering the mechanisms of astragaloside are important for the treatment of cardiovascular disease. Therefore, further in-depth investigations on astragaloside for cardiomyocyte hypertrophy are needed, especially at the molecular level, to provide a safer and more reliable treatment modality for cardiovascular diseases.

\section{References}

1. Bae, S., Singh, S. S., Yu, H., Lee, J. Y., Cho, B. R. and Kang, P. M. (2013). Vitamin D signaling pathway plays an 
important role in the development of heart failure after myocardial infarction. J Appl Physiol (1985), 114: 979-987.

2. Barker, T., Martins, T. B., Hill, H. R., Kjeldsberg, C. R., Dixon, B. M., Schneider, E. D., Henriksen, V. T. and Weaver, L. K. (2014). Vitamin D sufficiency associates with an increase in anti-inflammatory cytokines after intense exercise in humans. Cytokine, 65: 134-137.

3. Bavry, A. A., Handberg, E. M., Huo, T., Lerman, A., Quyyumi, A. A., Shufelt, C., Sharaf, B., Merz, C. N., Cooper-Dehoff, R. M., Sopko, G. and Pepine, C. J. (2014). Aldosterone inhibition and coronary endothelial function in women without obstructive coronary artery disease: an ancillary study of the national heart, lung, and blood institute-sponsored women's ischemia syndrome evaluation. Am Heart J, 167: 826-832.

4. Cohn, J. N. (2007). Reducing cardiovascular risk by blockade of the renin-angiotensin-aldosterone system. Adv Ther, 24: 1290-1304.

5. Colucci, W. S. (1997). Molecular and cellular mechanisms of myocardial failure. Am J Cardiol, 80: 15L-25L.

6. Devereux, R. B., Savage, D. D., Sachs, I. and Laragh, J. H. (1983). Relation of hemodynamic load to left ventricular hypertrophy and performance in hypertension. Am J Cardiol, 51: 171-176.

7. Ding, L., Dong, L., Chen, X., Zhang, L., Xu, X., Ferro, A. and Xu, B. (2009). Increased expression of integrin-linked kinase attenuates left ventricular remodeling and improves cardiac function after myocardial infarction. Circulation, 120: 764-773.

8. Ehses, W., Niklaus, K., Brockmann, M., Angenendt, W. and Saborowski, F. (2000). Fatal arrhythmia in a juvenile athlete due to myocardial hypertrophy and infarction. Int J Sports Med, 21: 536-539.

9. Ferder, M., Inserra, F., Manucha, W. and Ferder, L. (2013). The world pandemic of vitamin D deficiency could possibly be explained by cellular inflammatory response activity induced by the renin-angiotensin system. Am J Physiol Cell Physiol, 304: C1027-1039.

10. Glenn, D. J., Cardema, M. C. and Gardner, D. G. (2016). Amplification of lipotoxic cardiomyopathy in the VDR gene knockout mouse. J Steroid Biochem Mol Biol, 164: 292-298.

11. Guan, F. Y., Yang, S. J., Liu, J. and Yang, S. R. (2015). Effect of astragaloside IV against rat myocardial cell apoptosis induced by oxidative stress via mitochondrial ATP-sensitive potassium channels. Mol Med Rep, 12: 371-376.

12. Hu, L. W., Liberti, E. A. and Barreto-Chaves, M. L. (2005). Myocardial ultrastructure in cardiac hypertrophy induced by thyroid hormone--an acute study in rats. Virchows Arch, 446: 265-269.

13. Huang, H., Lai, S., Wan, Q., Qi, W. and Liu, J. (2016). Astragaloside IV protects cardiomyocytes from anoxia/reoxygenation injury by upregulating the expression of Hes1 protein. Can J Physiol Pharmacol, 94: 542-553.

14. Karpanen, T., Bry, M., Ollila, H. M., Seppanen-Laakso, T., Liimatta, E., Leskinen, H., Kivela, R., Helkamaa, T., Merentie, M., Jeltsch, M., Paavonen, K., Andersson, L. C., Mervaala, E., Hassinen, I. E., Yla-Herttuala, S., Oresic, M. and Alitalo, K. (2008). Overexpression of vascular endothelial growth factor-B in mouse heart alters cardiac lipid metabolism and induces myocardial hypertrophy. Circ Res, 103: 1018-1026.

15. Lee, T. W., Lee, T. I., Chang, C. J., Lien, G. S., Kao, Y. H., Chao, T. F. and Chen, Y. J. (2015). Potential of vitamin D in treating diabetic cardiomyopathy. Nutr Res, 35: 269-279.

16. Li, L., Hou, X., Xu, R., Liu, C. and Tu, M. (2017). Research review on the pharmacological effects of astragaloside IV. Fundam Clin Pharmacol, 31: 17-36.

17. Li, Y. C., Kong, J., Wei, M., Chen, Z. F., Liu, S. Q. and Cao, L. P. (2002). 1,25-Dihydroxyvitamin D(3) is a negative endocrine regulator of the renin-angiotensin system. J Clin Invest, 110: 229-238.

18. Liu, L. C., Voors, A. A., Van Veldhuisen, D. J., Van Der Veer, E., Belonje, A. M., Szymanski, M. K., Sillje, H. H., Van 286 
Gilst, W. H., Jaarsma, T. and De Boer, R. A. (2011). Vitamin D status and outcomes in heart failure patients. Eur J Heart Fail, 13: 619-625.

19. Lother, A. and Hein, L. (2016). Pharmacology of heart failure: From basic science to novel therapies. Pharmacol Ther, 166: 136-149.

20. Lu, S., Zhang, J. and Yang, D. (1999). [Effects of Astragaloside in treating myocardial injury and myocardial Sarco/Endoplasmic Ca(2+)-ATPase of viral myocarditis mice]. Zhongguo Zhong Xi Yi Jie He Za Zhi, 19: 672-674.

21. Luo, H. M., Dai, R. H. and Li, Y. (1995). [Nuclear cardiology study on effective ingredients of Astragalus membranaceus in treating heart failure]. Zhongguo Zhong Xi Yi Jie He Za Zhi, 15: 707-709.

22. Mahdy Ali, K., Wonnerth, A., Huber, K. and Wojta, J. (2012). Cardiovascular disease risk reduction by raising HDL cholesterol--current therapies and future opportunities. Br J Pharmacol, 167: 1177-1194.

23. Menaouar, A., Florian, M., Wang, D., Danalache, B., Jankowski, M. and Gutkowska, J. (2014). Anti-hypertrophic effects of oxytocin in rat ventricular myocytes. Int J Cardiol, 175: 38-49.

24. Meng, D., Chen, X. J., Bian, Y. Y., Li, P., Yang, D. and Zhang, J. N. (2005). Effect of astragalosides on intracellular calcium overload in cultured cardiac myocytes of neonatal rats. Am J Chin Med, 33: 11-20.

25. Mukherjee, A., Zerwekh, J. E., Nicar, M. J., Mccoy, K. and Buja, L. M. (1981). Effect of chronic vitamin D deficiency on chick heart mitochondrial oxidative phosphorylation. J Mol Cell Cardiol, 13: 171-183.

26. Muscogiuri, G., Annweiler, C., Duval, G., Karras, S., Tirabassi, G., Salvio, G., Balercia, G., Kimball, S., Kotsa, K., Mascitelli, L., Bhattoa, H. P. and Colao, A. (2016). Vitamin D and cardiovascular disease: From atherosclerosis to myocardial infarction and stroke. Int J Cardiol,230:577.

27. Nakane, M., Ma, J., Ruan, X., Kroeger, P. E. and Wu-Wong, R. (2007). Mechanistic analysis of VDR-mediated renin suppression. Nephron Physiol, 107: p35-44.

28. Pilz, S., Verheyen, N., Grubler, M. R., Tomaschitz, A. and Marz, W. (2016). Vitamin D and cardiovascular disease prevention. Nat Rev Cardiol, 13: 404-417.

29. Pourdjabbar, A., Dwivedi, G. and Haddad, H. (2013). The role of vitamin D in chronic heart failure. Curr Opin Cardiol, 28: $216-222$.

30. Saris, J. J., T Hoen, P. A., Garrelds, I. M., Dekkers, D. H., Den Dunnen, J. T., Lamers, J. M. and Jan Danser, A. H. (2006). Prorenin induces intracellular signaling in cardiomyocytes independently of angiotensin II. Hypertension, 48: 564-571.

31. Sciarretta, S., Paneni, F., Palano, F., Chin, D., Tocci, G., Rubattu, S. and Volpe, M. (2009). Role of the renin-angiotensin-aldosterone system and inflammatory processes in the development and progression of diastolic dysfunction. Clin Sci (Lond), 116: 467-477.

32. Shi, H., Ma, C., Liu, Y., Zhou, J., Hu, Z. and Wu, D. (2009). [Inhibitory effect on activated renin-angiotensin system by astragaloside IV in rats with pressure-overload induced cardiac hypertrophy]. Zhongguo Zhong Yao Za Zhi, 34 : 3242-3246.

33. Wang, Q., Xie, A., Xu, H. and Yu, J. (2016). OS 36-02 Astragaloside IV ameliorated mitochondrial oxidative stress in hypertensive mice with cardiomyopathy by regulating mitochondrial $\mathrm{Ca}^{2+}$ - flux. J Hypertens, 34 Suppl 1: e402.

34. Wang, S. G., Xu, Y., Xie, H., Wang, W. and Chen, X. H. (2015). Astragaloside IV prevents lipopolysaccharide-induced injury in H9C2 cardiomyocytes. Chin J Nat Med, 13: 127-132.

35. Weir, M. R. and Dzau, V. J. (1999). The renin-angiotensin-aldosterone system: a specific target for hypertension management. Am J Hypertens, 12: 205S-213S

36. Xiang, W., Kong, J., Chen, S., Cao, L. P., Qiao, G., Zheng, W., Liu, W., Li, X., Gardner, D. G. and Li, Y. C. (2005). 
Chen et al., Afr J Tradit Complement Altern Med., (2017) 14 (4): 278-288

\section{https://doi.org/10.21010/ajtcam.v14i4.31}

Cardiac hypertrophy in vitamin D receptor knockout mice: role of the systemic and cardiac renin-angiotensin systems. Am J Physiol Endocrinol Metab, 288: E125-132.

37. Xie, W. and Du, L. (2011). Diabetes is an inflammatory disease: evidence from traditional Chinese medicines. Diabetes Obes Metab, 13: 289-301.

38. Yang, J., Wang, H. X., Zhang, Y. J., Yang, Y. H., Lu, M. L., Zhang, J., Li, S. T., Zhang, S. P. and Li, G. (2013). Astragaloside IV attenuates inflammatory cytokines by inhibiting TLR4/NF-small ka, CyrillicB signaling pathway in isoproterenol-induced myocardial hypertrophy. J Ethnopharmacol,150(3):1062.

39. Yao, T., Ying, X., Zhao, Y., Yuan, A., He, Q., Tong, H., Ding, S., Liu, J., Peng, X., Gao, E., Pu, J. and He, B. (2015). Vitamin D receptor activation protects against myocardial reperfusion injury through inhibition of apoptosis and modulation of autophagy. Antioxid Redox Signal, 22: 633-650.

40. Yoshida, S., Ishizawa, K., Ayuzawa, N., Ueda, K., Takeuchi, M., Kawarazaki, W., Fujita, T. and Nagase, M. (2014). Renin inhibition ameliorates renal damage through prominent suppression of both angiotensin I and II in human renin angiotensinogen transgenic mice with high salt loading. Clin Exp Nephrol, 18: 593-599.

41. Zhang, S., Tang, F., Yang, Y., Lu, M., Luan, A., Zhang, J., Yang, J. and Wang, H. (2015). Astragaloside IV protects against isoproterenol-induced cardiac hypertrophy by regulating NF-kappaB/PGC-1alpha signaling mediated energy biosynthesis. PLoS One, 10: e0118759.

42. Zhou, C., Lu, F., Cao, K., Xu, D., Goltzman, D. and Miao, D. (2008). Calcium-independent and 1,25(OH)2D3-dependent regulation of the renin-angiotensin system in 1alpha-hydroxylase knockout mice. Kidney Int, 74: 170-179. 